\title{
Does the substitution of cisplatin in a standard four drug regimen improve survival in small cell carcinoma of the lung? A comparison of two chemotherapy regimens
}

\author{
A P Smith, G Anderson, G Chappell, D R Bowen
}

\begin{abstract}
Ninety five patients with small cell carcinoma of the lung were randomly assigned to one of two chemotherapy regimens (VACE or CVACE), each consisting of six cycles at three week intervals. The VACE regimen consisted of six cycles of vincristine $1.2 \mathrm{mg} / \mathrm{m}^{2}$, doxorubicin $40 \mathrm{mg} / \mathrm{m}^{2}$, and cyclophosphamide $700 \mathrm{mg} / \mathrm{m}^{2}$ on day 1 plus etoposide $110 \mathrm{mg} / \mathrm{m}^{2}$ daily for three days. The CVACE regimen was identical to the VACE regimen for cycles 3 and 4; cycles $1,2,4$, and 6 consisted of etoposide $110 \mathrm{mg} / \mathrm{m}^{2}$ for three days plus cisplatin $100 \mathrm{mg} / \mathrm{m}^{2}$ with mannitol diuresis on the second day. Forty eight patients received VACE and 47 CVACE. Side effects resulted in withdrawal of four patients receiving VACE and six receiving CVACE. Three deaths were attributed to VACE and one to CVACE. Median survival did not differ between the two treatments overall, though there was a small increase in median survival in partial responders receiving CVACE. It is concluded that replacing four of the six cycles of VACE (vincristine, doxorubicin, cyclophosphamide, and etoposide) with etoposide and cisplatin conferred no overall advantage.
\end{abstract}

Chemotherapy protocols that include platinum compounds are widely used for the treatment of small cell lung cancer and in some instances have been claimed to increase survival. ${ }^{1}$ We have reported encouraging results from an alternating treatment protocol containing cisplatin in an uncontrolled study. ${ }^{2}$ We now report the results of a controlled trial of a similar chemotherapy regimen. Although cisplatin has been used successfully in combination with other drugs in small cell lung cancer, it is relatively expensive and toxic (causing especially nausea and vomiting), and because of the risk of nephrotoxicity patients require hospital admission to ensure proper hydration and adequate urinary flow for each treatment. We wished to establish whether the addition of cisplatin in an alternating chemotherapy protocol improved the outcome for patients with small cell lung cancer.

\section{Methods}

Ninety five patients (33 female) with histologically confirmed small cell lung cancer entered the study. The study protocol was approved by the local ethical committees.

Before treatment the extent of the disease was staged on the basis of the history, clinical examination, chest radiograph, bronchoscopy findings, ultrasound liver scan, blood count, and serum biochemistry. Computed tomography and isotope scanning of liver, bone or brain were performed when appropriate and in the event of equivocal results from clinical, biochemical or radiological investigations.

Disease was considered to be "limited" if confined to one hemithorax, with or without disease of the ipsilateral supraclavicular lymph nodes, and "extensive" when tumour had spread beyond these sites.

\section{PROTOCOL}

Patients were randomly allocated to two groups stratified for age, sex, and stage of disease. The first received six cycles of the VACE combination-vincristine $1.2 \mathrm{mg} / \mathrm{m}^{2}$, doxorubicin $40 \mathrm{mg} / \mathrm{m}^{2}$, and cyclophosphamide $700 \mathrm{mg} / \mathrm{m}^{2}$ on day 1 , plus etoposide $110 \mathrm{mg} / \mathrm{m}^{2}$ daily for three days; this cycle was repeated at three weekly intervals. The second group received the CVACE combinationetoposide $110 \mathrm{mg} / \mathrm{m}^{2}$ for three days plus cisplatin $100 \mathrm{mg} / \mathrm{m}^{2}$ with mannitol diuresis on the second day for the first two three weekly cycles. VACE in the above doses was administered for the next two cycles; cycles 5 and 6, if given, consisted of etoposide and cisplatin in the same doses as before. The effects of treatment were assessed formally at the end of cycle 4. If a complete or partial remission had been obtained a further two cycles were given. Patients who had not responded were withdrawn three weeks after cycle four (that is, when cycle five was due) and considered for alternative treatment, which in most cases consisted of radiotherapy. Complete responders after cycle 6 were randomly allocated to a group receiving prophylactic cranial irradiation and to a no treatment group. Partial responders received further chemotherapy (with mitomycin $C$ and procarbazine) or radiotherapy or no further treatment depending on individual circumstances.

Dose modifications were made in the event 
Table 1 Clinical details of the patients

\begin{tabular}{lll}
\hline & $V A C E$ & $C V A C E$ \\
\hline No of patients & 48 & 47 \\
Mean age (range): y & $61(37-75)$ & $60(46-72)$ \\
Male:female & $27: 21$ & $35: 12$ \\
No (\%) with extensive disease & $18(38)$ & $17(36)$ \\
\hline
\end{tabular}

VACE-group receiving vincristine, doxorubicin, cyclophosphamide, and etoposide; CVACE-group receiving VACE with cisplatin in place of vincristine, doxorubicin, and cyclophosphamide in some cycles (see under "Protocol").

of haematological impairment or renal failure as necessary. Side effects were recorded at each visit. Withdrawals from the trial were permitted at any time, the reasons for withdrawal being stated.

Complete remission was defined as the complete disappearance of all lesions for four or more weeks from cessation of treatment, and partial remission as a reduction by at least half in the sum of the two greatest diameters of a measurable lesion for four weeks. Patients were followed up at monthly intervals for six months and at three monthly intervals thereafter.

\section{STATISTICAL ANALYSIS}

Analysis of results was performed with life tables and the log rank test. ${ }^{34}$ Confidence limits for median survival were obtained from the survival curves by the method of Simon. ${ }^{5}$

\section{Results}

Clinical details of the patients are shown in table 1 .

Ninety five patients entered the study, of whom 47 were randomised to receive CVACE and 48 VACE; of these patients, 40 and 38 respectively were given four or more cycles of treatment.

\section{WITHDRAWALS}

Of the 17 patients receiving less than four cycles, five had received VACE, three having

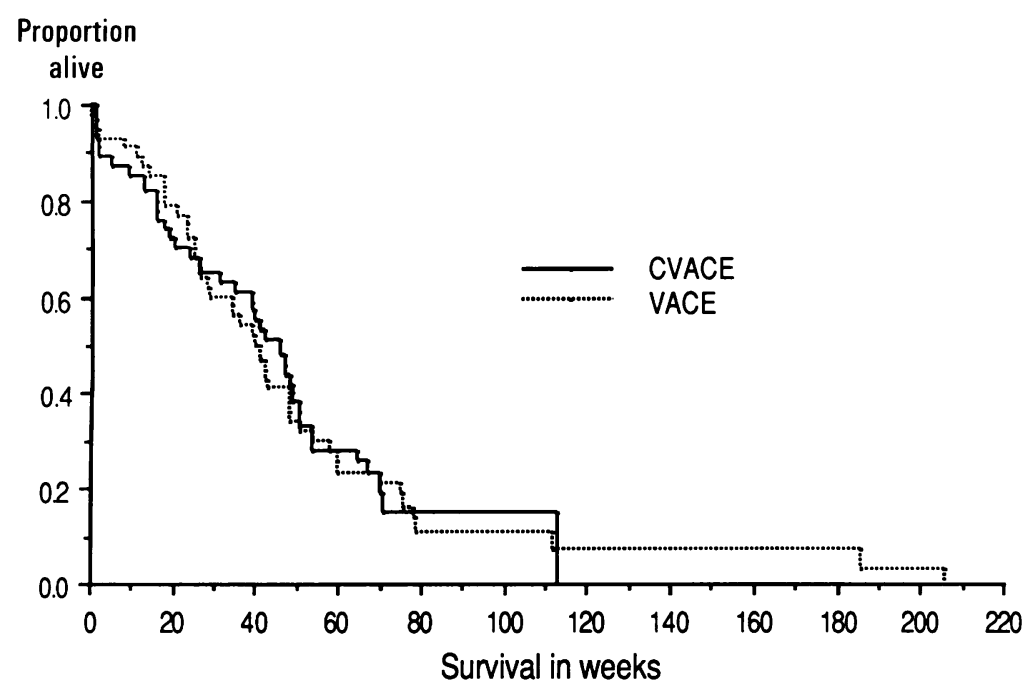

Survival curves comparing patients receiving VACE (vincristine, doxorubicin, cyclophosphamide, and etoposide) with those receiving CVACE (cisplatin used in some cycles in place of vincristine, doxorubicin, and cyclophosphamide-see under "Protocol").
Table 2 Response to treatment

\begin{tabular}{lll}
\hline & VACE & CVACE \\
\hline No (\%) with & & \\
complete remission & $21(44)$ & $23(49)$ \\
partial remission & $10(21)$ & $11(23)$ \\
no remission & $17(35)$ & $13(28)$ \\
\hline
\end{tabular}

Abbreviations as in table 1

received three cycles. Four were withdrawn because of leucopenia and one because of severe vomiting. Of the six withdrawals of patients receiving CVACE, one was because of renal failure, four were due to nausea and vomiting or leucopenia (all after one or two cycles of treatment), and one was because cerebral metastases developed after two cycles of treatment. Six patients died from lung cancer before they had received three cycles of VACE, and one patient died at home (cause unknown) after one cycle of CVACE. All these patients were included in the analysis.

DEATHS RELATED TO TREATMENT

Three deaths were attributed to treatment with VACE, all due to leucopenia with severe pulmonary infection. One patient receiving CVACE died of renal failure.

\section{RESPONSE}

Responses to treatment are displayed in table 2. There was no difference in response rates between the two treatment regimens. Nineteen patients with limited disease did not respond to treatment, of whom 11 had received VACE and eight CVACE.

\section{SURVIVAL}

The median survival was 47 (95\% confidence limits 35-50) weeks for patients receiving VACE and 40 (95\% CL 28-47) weeks for those receiving CVACE. The difference was not statistically significant (figure). In patients with a complete remission median survival was 54 weeks for both regimens, compared with 16 and 18 weeks for VACE and CVACE for the patients showing no response. Patients with a partial remission lived longer if they had received VACE (median survival 48 (95\% CL 39-66) weeks) than if they had received CVACE (28 (95\% CL 23-48) weeks$\mathrm{p}<0.005$.

There were no differences in median survival between treatments according to stage.

Eighteen patients with extensive disease obtained a complete remission, nine in each group; the median survival times were 67 weeks for VACE and 48 weeks for CVACE (difference not significant).

CAUSE OF DEATH

Seventy seven patients have died. With the exceptions of the causes mentioned above, all deaths were due to lung cancer. Of the 44 patients with a complete response to treatment, 14 are still alive; 14 died of isolated cranial metastases, five of widespread metastases including the brain, and 11 with widespread disease without brain metastases. In complete 
responders brain metastases were the most common cause of death.

EFFECT OF PROPHYLACTIC CRANIAL IRRADIATION Twelve complete responders received prophylactic cranial irradiation, six in each treatment group. Five subsequently died from cranial metastases, five are alive, one died from a local recurrence, and one died from unknown causes.

\section{Discussion}

Evidence that including cisplatin in treatment protocols improves the outcome of treatment for small cell lung cancer is scanty, though numerous publications report its use. ${ }^{16-10}$ In a series of previously treated patients who had relapsed Levinson et $a^{6}{ }^{6}$ reported only marginal benefit from cisplatin used on its own. Sculier et $a l,{ }^{8}$ using a regimen consisting of cisplatin, doxorubicin, vincristine, and etoposide for 10 cycles, report results almost identical to those we obtained ${ }^{2}$ with alternating regimens containing the same drug combinations. Alternating regimens comparing VAC with VAC plus cisplatin and etoposide ${ }^{11}$ indicated a modest but significant superiority of the alternating regimen containing cisplatin. Response rates in the present trial were $72 \%$ with CVACE and $64 \%$ with VACE. The difference was not significant but numbers are small and we may have missed a true treatment difference. Aisner $e t a l^{9}$ found that the addition of cisplatin to a standard regimen of doxorubicin, cyclophosphamide, and etoposide did not improve response, but there was a small improvement in survival. Our study showed no difference in response or survival overall; there was a small difference in favour of VACE in the partial responders but numbers were small. Patients in the CVACE limb of the trial did not receive doxorubicin or cyclophosphamide during four of the six cycles and we cannot say whether omission of these drugs affected survival.

Although drug combinations with cisplatin have been shown to be active in the treatment of small cell lung cancer, cisplatin itself has been disappointing. It is highly effective when given alone but has failed to enhance survival when combined with other active drugs. Its inclusion in induction treatment therefore may not be justified in view of the increased toxicity, especially renal toxicity, and its lack of impact on survival.
The number of patients receiving prophylactic cranial irradiation in this study was too small to permit any definite conclusion. Our results do not suggest that this approval is likely to be a solution to the widespread problem of death from cerebral metastases in patients with remissions that are otherwise complete.

Our results show that replacing VAC with cisplatin and etoposide for four out of six treatment cycles confers no advantage in terms of survival or of toxicity. The small differences we saw between the two treatment groups suggest that non-alternating VACE without cisplatin may be the better treatment. There is no justification for the increased expense and inconvenience of using cisplatin in induction treatment for small cell lung cancer.

We are grateful to our colleagues in South Wales who referred

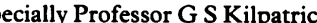
Dr B H Davies, Mr E G Butchart, Dr I S Petheram, and Dr Cotton. We thank Miss M S Brewer for typing the manuscript.

1 Evans WK, Shepherd FA, Feld R, Osoba D, Dang P, Deboer G. VP-16 and cisplatin as first line chemotherapy for small cell lung cancer. J Clin Oncol 1985;3:1471-7.

2 Littlewood TJ, Smith AP, Anderson G, Chappell AG James $\mathrm{KW}$. Cisplatin and etoposide alternating with vincristine, doxorubicin and cyclophosphamide in patients with small cell lung cancer. Eur $J$ Respir Dis 1985;67:294-300.

3 Peto R, Pike MC, Armitage P, et al. Design and analysis of randomised clinical trials requiring prolonged observation of each patient. I. Introduction and design. $\mathrm{Br} J$ Cancer 1976;34:585-612.

4 Peto R, Pike MC, Armitage P, et al. Design and analysis of randomised clinical trials requiring prolonged observation of each patient. II Analysis and examples. Br J Cancer 1977;35:1-39.

5 Simon R. Confidence limits for reporting results of clinical trials. Ann Intern Med 1986;105:429-35.

6 Levenson RM, Ihde DC, Huberman MS, Cohen MH, Bunn PA, Minna JD. Phase II Trial of cisplatin in small cell carcinoma of the lung. Cancer Treat Rep 1981;65:905-7.

7 Madrigal PA, Manga GP, Palomero I, Gomez RG. VP16 213 combined with cis-platinum (CDDP) in the treatment of small cell carcinoma of the lung. Cancer Chemother Pharmacol 1982;7:203-4.

8 Sculier JP, Klastersky J, Becquart D, et al. Phase II Study of an intensive combination chemotherapy with cisplatin, adriamycin, etoposide and cyclophosphamide (CAVE) in 519-26.

9 Aisner J, Whiteacre M, Abrams J, Propert K. Doxorubicin, cyclophosphamide, etoposide and platinum, doxorubicin, cyclophosphamide and etoposide for small cell carcinom of the lung. Semin Oncol 1986;13(suppl 3):54-62.

10 Evans WK, Feld R, Murray N, et al. The use of VP16 plus cisplatin during induction chemotherapy for small cell lung cancer. Semin Oncol 1986;13(suppl 3):10-6.

11 Evans WK, Feld R, Murray N, et al. Superiority of alternating non cross resistant chemotherapy in extensive small cell lung cancer. A multicentre, randomised clinical trial by the National Cancer Institute of Canada. Ann Intern Med 1987;107:451-8. 